\title{
Role of Cerebrospinal Fluid Pleocytosis and Haemophilus influenzae Type b Capsule on Blood Brain Barrier Permeability during Experimental Meningitis in the Rat
}

\author{
Alan J. Lesse, ${ }^{\star}$ E. Richard Moxon, ${ }^{\ddagger}$ André Zwahlen, and W. Michael Scheld*\| \\ Departments of Internal Medicine* and Neurosurgery," University of Virginia School of Medicine, Charlottesville, Virginia 22908; \\ Department of Pediatrics, ${ }^{\ddagger}$ Oxford University, Oxford, United Kingdom; and Department of Medicine, ${ }^{\S}$ Hopital de Zone \\ St. Loup/Orbe, Pompaples, Switzerland
}

\begin{abstract}
The influence of leukocytes and Haemophilus influenzae type b (Hib) capsule on blood brain barrier permeability (BBBP) to circulating ${ }^{125} \mathrm{I}$-albumin in normal and leukopenic rats was assessed after intracisternal inoculation of encapsulated $\left(\operatorname{Rd}^{-} /\right.$ $\left.b^{+} / 02\right)$ or unencapsulated $\left(\mathrm{Rd}^{-} / \mathrm{b}^{-} / 02\right)$ isogenic strains of $\mathrm{Hib}$. Both normal and leukopenic animals had increased BBBP $18 \mathrm{~h}$ after inoculation, with normal rats demonstrating significantly increased BBBP after challenge with the encapsulated strain. Despite cerebrospinal fluid (CSF) pleocytosis in normal rats, CSF bacterial concentrations were not lower. Normal rats cleared unencapsulated $\mathrm{Rd}^{-} / \mathrm{b}^{-} / 02$ more effectively than leukopenic rats, with BBBP correlating with CSF bacterial density and not leukocyte concentrations. Challenge with heatkilled $\mathrm{Rd}^{-} / \mathrm{b}^{+} / 02$ resulted in increased BBBP in both normal and leukopenic rats, with greater BBBP at higher bacterial concentrations. The data suggest: $(a)$ significant increases in BBBP occur in the near absence of CSF leukocytes; (b) CSF leukocytes can augment changes in BBBP; (c) type b capsule inhibits host clearance mechanisms within the CSF; and $(d)$ BBBP appears to correlate with bacterial concentrations within the CSF.
\end{abstract}

\section{Introduction}

Despite the introduction of newer antibiotics with greatly enhanced in vitro activity against the common meningeal pathogens, the mortality of bacterial meningitis has changed little over the last 30 years $(1,2)$. Inadequate knowledge of central nervous system (CNS) dynamics during bacterial meningitis has contributed to this lack of progress. Although it is well

This paper was presented in part at the 26th Interscience Conference on Antimicrobial Agents and Chemotherapy, in New Orleans, LA, October 1986 (abstract No. 747), and at the annual meeting of the Southern Society for Clinical Investigation, in New Orleans, LA, January 1987 (1987. Clin. Res. 41A [Abstr.]).

Address correspondence and reprint requests to Dr. Scheld, Depts. of Internal Medicine and Neurosurgery, Div. of Infectious Diseases, Box 385, University of Virginia School of Medicine, Charlottesville, VA 22908. Dr. Lesse's current address is Depts. of Medicine, Pharmacology, and Therapeutics, State University of New York at Buffalo, and Veteran's Administration Hospital, Buffalo, NY.

Received for publication 10 February 1987 and in revised form 22 January 1988.

J. Clin. Invest.

(C) The American Society for Clinical Investigation, Inc.

0021-9738/88/07/0102/08 \$2.00

Volume 82, July 1988, 102-109 recognized that the CNS is an area of impaired host resistance, a clearer understanding of the pathophysiologic changes that occur during meningitis is mandatory if improvements in outcome from this infection are to occur.

One of the prominent pathophysiologic alterations in the CNS during bacterial meningitis is the development of a profound leukocytosis in the cerebrospinal fluid (CSF). ${ }^{1}$ Despite its early historical recognition (3), the role of the CSF leukocyte remains unclear. Conflicting data exist regarding the function of the leukocytes in the CSF during bacterial meningitis. While CSF leukocytes may be beneficial in eradicating microorganisms from the subarachnoid space, several studies have suggested that the influx of leukocytes into the CNS may be accompanied by deleterious alterations of CSF hydrodynamics, brain metabolism, and CNS homeostasis (4-11).

Although it is postulated that the CSF leukocytosis may potentially injure the host, it is a widely held belief that the influx of leukocytes into the CSF serves to limit the bacterial replication of organisms within the CSF. The mechanism that most readily accomplishes this function outside the CNS is phagocytosis. However, in the peripheral circulation phagocytosis is greatly aided by the presence of opsonizing antibodies and complement, two components that are virtually absent from normal CSF $(12,13)$.

The major meningeal pathogens, Haemophilus influenzae type b, Streptococcus pneumoniae, and Neisseria meningitidis, all possess cell surface polysaccharide capsules that impede phagocytosis in the absence of opsonizing factors. Capsular polysaccharides would therefore appear to limit further the effectiveness of CSF leukocytes, but a direct experimental relationship between capsular presence and bacterial clearance in the CNS has not been established rigorously. Previous comparisons of encapsulated and unencapsulated bacteria contrasted the virulence of bacterial strains that differed not only with respect to capsule but with regard to lipopolysaccharide, outer membrane proteins, and other surface characteristics, and could not specifically implicate capsular determinants in the disease process. The recent availability of isogenic bacterial strains of $H$. influenzae type b overcomes these difficulties and has made it possible to investigate the interplay of leukocytes and capsule in the pathophysiologic consequences of meningitis.

Previous work from this laboratory has shown that meningitis results in profound ultrastructural changes in the major anatomical site of the blood brain barrier (BBB), the cerebral

1. Abbreviations used in this paper: BBB, blood brain barrier; BBBP, blood brain barrier permeability; CSF, cerebrospinal fluid; Hib, Haemophilus influenzae type b; LOS, lipooligosaccharide; WBC, white blood cell. 
capillary endothelium (14). Increased pinocytotic vesicles and separation of tight junctional complexes in cerebral capillary endothelium were seen to increase temporally during meningitis, and these ultrastructural changes correlated well with the entry of circulating ${ }^{125} \mathrm{I}$-albumin into the CSF, a sensitive measurement of functional BBB injury. This study (14) helped define the changes in the BBB during meningitis but did not address the role of the CSF leukocyte during the infection.

To examine the role of CSF leukocytes and $H$. influenzae type $b$ capsule during bacterial meningitis, we used a model of leukopenia in the adult rat to assess the impact of leukocytes on the integrity of the BBB to circulating ${ }^{125} \mathrm{I}$-albumin. Functional integrity of the BBB in the presence of CSF leukocytes was also examined through the use of isogenic strains of $H$. influenzae type b to examine: (a) the relationship of leukocyte function and capsular polysaccharide within both the CSF and the bloodstream; $(b)$ the role of the antiphagocytic properties of type b capsular polysaccharide; and $(c)$ the influence of the interaction of leukocytes and capsule on the permeability of the $\mathrm{BBB}$ to circulating albumin.

\section{Methods}

Bacteria. The isogenic strains of $H$. influenzae used in this study were chosen to allow an unambiguous analysis of the role of type b capsule in mediating changes in blood brain barrier permeability (BBBP) during experimental meningitis in normal and leukopenic rats. $\mathrm{Rd}^{-} / \mathrm{b}^{+} / 02$ was obtained by transformation of strain Rd using donor DNA from strain Eagan (type b). $\mathbf{R d}^{-} / \mathrm{b}^{-} / 02$ is a spontaneous, one step, capsuledeficient mutant derived from $\mathrm{Rd}^{-} / \mathrm{b}^{+} / 02$. $\mathrm{Rd}^{-} / \mathrm{b}^{-} / 02$ does not make any polyribosyl-ribitol phosphate capsule (by immunofluorescence [15] or ELISA, with a lower limit of dectability of $0.1 \mathrm{ng} / \mathrm{ml}$ [16]) because it has a mutation in a defined locus within the chromosomal region required for type $b$ capsular expression (17). Additional experiments also utilized another $H$. influenzae type b (strain Eagan), which has been shown to produce meningitis in experimental animals (especially infant rats) and is the classic strain used in those models.

The transformants $\left(\mathrm{Rd}^{-} / \mathrm{b}^{+} / 02\right.$ and $\left.\mathrm{Rd}^{-} / \mathrm{b}^{-} / 02\right)$ possessed identical lipooligosaccharide (LOS) patterns on SDS-PAGE. In addition, similar outer membrane protein patterns are observed (data not shown). Strain Eagan possessed a different LOS pattern than the one seen in the transformants by SDS-PAGE. Transformation of the strain Rd with Eagan DNA results in altered LOS patterns from donor to recipient (18). The LOS of $\mathrm{Rd}^{-} / \mathrm{b}^{+} / 02$ and Eagan also differ antigenically as assessed by identification with $H$. influenzae type b LOS-specific murine monoclonal antibodies (reactive with epitopes in the oligosaccharide side chain) provided by Dr. Eric Hansen (Dallas, TX) $(19,20)$. The Eagan strain is reactive with both monoclonal antibodies $(12 \mathrm{D} 9+, 4 \mathrm{C} 4+)$ in a colony blot radioimmunoassay and is antigenically placed in group 3 of a recent classification scheme (20). $\mathrm{Rd}^{-} / \mathrm{b}^{+} /$ 02 and $\mathrm{Rd}^{-} / \mathrm{b}^{-} / 02$, both $12 \mathrm{D} 9+, 4 \mathrm{C} 4-$, are placed in group 1 , and are identical by monoclonal antibody analysis of LOS. Exhaustive structural analysis of the precise differences between $\mathrm{Rd}^{-} / \mathrm{b}^{+} / 02$ and Eagan is in progress.

The bacteria were grown in brain heart infusion broth (Gibco, Grand Island, NY) with 5\% Fildes supplement (Difco Laboratories, Detroit, MI) to late $\log$ phase then frozen in skim milk at $-70^{\circ} \mathrm{C}$ until use. For each experiment, a vial was thawed and grown overnight in brain heart infusion broth with 5\% Fildes enrichment. To obtain the final inoculum this overnight growth was inoculated $(10 \mu \mathrm{l})$ into 10 $\mathrm{cm}^{3}$ brain heart infusion broth with 5\% Fildes and grown for $8 \mathrm{~h}$, which is when the organisms were in late log phase growth and expressing maximal capsule. The broth was spun at $2,500 \mathrm{~g}$ for $15 \mathrm{~min}$ at $20^{\circ} \mathrm{C}$ and the pellet was washed with $10 \mathrm{ml}$ of $0.9 \%$ sodium chloride solution and centrifuged at $2,500 \mathrm{~g}$ twice. The pellet was then suspended in saline to a concentration of $\sim 10^{8} \mathrm{CFU} / \mathrm{ml}$ as determined by plating of 100-fold serial dilutions of the organisms on chocolate agar (BBL, Cockesville, MD). This concentration resulted in an inoculum of $10^{6-6.5} \mathrm{CFU}$ per animal. The centrifugation and washing procedures removed $\leq 5 \%$ of the capsule from the encapsulated organisms.

Heat-killed bacteria were obtained by heating the initial and final dilution of organisms to $65^{\circ} \mathrm{C}$ for $30 \mathrm{~min}$ in a heating block. Efficiency of the procedure was confirmed by subculture of the inocula (demonstrating complete sterility). Animals received either $\approx 10^{6.5}$ or $\approx 10^{8.5}$ CFU. Scanning electron micrographs revealed no discernible change in the live versus heat-killed bacteria (data not shown).

We sought to develop a model of sterile pleocytosis to better define the relationship between BBBP and CSF pleocytosis without the confounding variable of bacterial products in the CSF. Multiple compounds were injected intracisternally to achieve a nonbacterial model of sterile pleocytosis without success. These included $f$ Met-Leu-Phe and its active esters, mucin, $\mathrm{CS}_{\mathrm{a}}$, cobra venom factor, and alpha bungarotoxin. These substances either resulted in no CSF pleocytosis or were too toxic for the host.

Production of leukopenia. Leukopenia was induced in 125-200-g Wistar rats by the intraperitoneal injection of cyclophosphamide (Adria Laboratories, Columbus, $\mathrm{OH}$ ) at a dose of $100 \mathrm{mg} / \mathrm{kg}$. The animals were then studied $4 \mathrm{~d}$ later when the total peripheral leukocyte concentration had fallen below 1,000 cells $/ \mathrm{mm}^{3}$ (nadir).

Induction of meningitis. We used our well-characterized model of meningitis in the adult rat (14) with minor modifications as outlined below. Briefly, 125-200-g Wistar rats (Hilltop Farms, Scottsdale, PA) were anesthetized with ketamine (Parke-Davis, Morris Plain, NJ), 100 $\mathrm{mg} / \mathrm{kg}$, and xylazine (Miles Laboratories, Swanee, KA), $7 \mathrm{mg} / \mathrm{kg}$, intramuscularly; repeated injections were given when necessary. The animals were shaved over the dorsal surface of the neck and occiput. Before the inoculation of the challenge strains, all animals had peripheral blood white blood cell (WBC) concentrations and platelet concentrations evaluated via cardiac puncture using unipettes (BBL). Additionally, $0.1 \mathrm{ml}$ of blood was plated on chocolate agar in all leukopenic animals to ensure that the rats were not bacteremic before the experiment.

The animals were then inoculated intracisternally via a percutaneous injection with a 25-gauge butterfly needle (Abbott Inc., North Chicago, IL) fitted to a micromanipulator. After removal of 50-75 $\mu \mathrm{l}$ of CSF, $50 \mu \mathrm{l}$ of the above described inoculum ( $\left.\sim 10^{6} \mathrm{CFU}\right)$ was injected directly into the cisterna magna. Control animals were given an equal volume of pyrogen-free saline intracisternally instead of the challenge strain. Only inoculations that were clear without any visible blood contamination were used. Leukopenic animals had $25 \mu \mathrm{l}$ of CSF cultured to confirm a sterile CSF at the initiation of the experiment. Quantitative CSF sampling was accomplished using 25- $\mu$ l fixed volume micropipettes (Dade Scientific, Miami, FL). The animals were allowed to progress with their infections until $18 \mathrm{hr}$ postinoculation. This time point results in a severe meningitis characterized by near maximal CSF inflammation in this model (14). At this time the rats were again anesthetized and CSF sampled via cisternal puncture. Again, if any contamination with blood was seen the animals were excluded from analysis. $25 \mu$ each of CSF were used for culture, WBC determination, and evaluation of BBBP (see below). Quantitative cultures of CSF and blood were determined by serial dilution in saline and culture on chocolate agar. Both blood and CSF WBC and platelet concentrations were determined in a hemocytometer by standard protocol. Differential cell counts (polymorphonuclear vs. mononuclear) were assessed on both CSF and blood from leukopenic rats; nonleukopenic CSF consistently contained $\geq 90 \%$ polymorphonuclear leukocytes.

The experiments were performed in lots of 8 to 10 animals. The values expressed are the averages for each respective group with $n$ determined as the total number of animals in the given group. Animals were excluded from data analysis that had: $(a)$ any blood contamination during CSF removal; $(b)$ death before $18 \mathrm{~h}$ postinoculation; or $(c)$ 
peripheral leukocyte concentrations $>1,000$ cells $/ \mathrm{mm}^{3}$ in the leukopenic group before inoculation.

Assessment of $B B B P .30 \mathrm{~min}$ after the intracisternal inoculation of the challenge organism, each animal was given $\approx 15 \mu \mathrm{Ci}$ of ${ }^{125}$ I-labeled bovine serum albumin (ICN, Irvine, $C A$ ) via intracardiac injection. At $18 \mathrm{~h}$, when the CSF was sampled, $25 \mu \mathrm{l}$ of CSF was collected in the micropipette and placed into a 1 dram vial. $25 \mu$ l of blood was also placed in a pipette and the samples were counted in a Gamma 300 counter (Beckman Instruments Inc., Irvine, $\mathrm{CA}$ ) for $10 \mathrm{~min}$. The permeability of the BBB was assessed as the $\mathrm{cpm}$ CSF/cpm blood $\times 100$ and expressed as a percentage.

Analysis. Statistical comparisons between groups were done by Student's $t$ test and $P$ values of $\leq 0.05$ were considered significant.

\section{Results}

The cyclophosphamide-induced leukopenic rat model resulted in a profound peripheral leukopenia. All leukopenic animals had peripheral WBC concentrations $<1,000$ cells $/ \mathrm{mm}^{3}$ with a mean \pm SEM concentration of $0.32 \pm 0.03 \times 10^{3} / \mathrm{mm}^{3}$. This is in sharp contrast with the normal rats who demonstrated peripheral mean $\pm S E M$ cell concentrations of $10.89 \pm 0.57$ $\times 10^{3} / \mathrm{mm}^{3}$.

In addition to the profound leukopenia, cyclophosphamide also produced a marked alteration in platelet concentrations. The mean \pm SEM platelet concentration for all normal animals before infection was $935 \pm 36 \times 10^{3} / \mathrm{mm}^{3}$, while leukopenic rats had a significantly lower mean \pm SEM platelet concentration of $361 \pm 19 \times 10^{3} / \mathrm{mm}^{3}(P<0.001)$. At $18 \mathrm{~h}$ after infection normal animals had a mean $\pm S E M$ platelet concentration of $806 \pm 16 \times 10^{3} / \mathrm{mm}^{3}$ compared with leukopenic animals which had a mean \pm SEM platelet concentration of $126 \pm 17 \times 10^{3} / \mathrm{mm}^{3}(P<0.001)$. No leukopenic rat ever had $<20,000$ platelets $/ \mathrm{mm}^{3}$.

The profound peripheral leukopenia also resulted in a marked decrease in CSF leukocytosis in all leukopenic rats studied. Table I shows the CSF cell concentrations of both normal and leukopenic animals. No rat in the leukopenic group ever had $>350$ cells $/ \mathrm{mm}^{3}$ of CSF (the average polymorphonuclear cell count was only $11 \%$ in the CSF from leukopenic rats) while the normal groups developed mean CSF WBC concentrations $>6,800$ cells $/ \mathrm{mm}^{3}$. The CSF pleocytosis was almost 16,000 cells $/ \mathrm{mm}^{3}$ after challenge with $\mathrm{Rd}^{-} / \mathrm{b}^{-} / 02$ (more than twofold greater than after inoculation with $\mathrm{Rd}^{-} / \mathrm{b}^{+} / 02$ ), yet leukopenic rats given the same organism demonstrated a mean CSF pleocytosis of only 90 cells $/ \mathrm{mm}^{3}$.

Blood bacterial concentrations were approximately $10^{6}$ $\mathrm{CFU} / \mathrm{ml}$ in the $\mathrm{Rd}^{-} / \mathrm{b}^{+} / 02$ group, with no difference seen between the leukopenic and normal animals (Table II). All animals inoculated with the encapsulated organisms were bacteremic. The unencapsulated organism was never present in the

Table I. CSF Leukocyte Concentrations $18 \mathrm{~h}$ after Intracisternal Inoculation

\begin{tabular}{crc}
\hline Inoculated strain & Normal rats* $(n)$ & Leukopenic rats* $(n)$ \\
\hline $\mathrm{Rd}^{-} / \mathrm{b}^{+} / 02$ & $6.8 \pm 1.6(12)$ & $0.14 \pm 0.03(13)$ \\
$\mathrm{Rd}^{-} / \mathrm{b}^{-} / 02$ & $15.9 \pm 2.6(11)$ & $0.09 \pm 0.02(12)$
\end{tabular}

${ }^{*}$ Mean \pm SEM leukocytes $\times 10^{3} / \mathrm{mm}^{3}$.
Table II. Blood Bacterial Concentrations 18 h after Intracisternal Inoculation

\begin{tabular}{|c|c|c|}
\hline Inoculated strain & Normal rats* $(n)$ & Leukopenic rats* $(n)$ \\
\hline $\mathrm{Rd}^{-} / \mathrm{b}^{+} / 02$ & $6.43 \pm 0.37(12)$ & $6.02 \pm 0.21(13)$ \\
\hline $\mathrm{Rd}^{-} / \mathrm{b}^{-} / 02$ & $0 \quad(11)$ & $2.32 \pm 0.41(6 / 12)^{\ddagger}$ \\
\hline
\end{tabular}

* Mean \pm SEM $\log _{10} \mathrm{CFU} / \mathrm{ml}$ blood.

‡ Only 6/12 animals were bacteremic. Value given is the mean \pm SEM of the six bacteremic rats.

blood of the normal animals but was detected in 6/12 leukopenic rats inoculated with this organism.

Despite the marked differences in the peripheral and CSF leukocyte concentrations between normal and leukopenic rats, the bacterial concentrations in the CSF of animals inoculated with the encapsulated organisms were similar (Table III). In fact, CSF concentrations of bacteria were higher in the normal rats inoculated with $\mathrm{Rd}^{-} / \mathrm{b}^{+} / 02$ when compared with the leukopenic rats $(P=0.005)$.

CSF bacterial concentrations of the unencapsulated strain $\mathrm{Rd}^{-} / \mathrm{b}^{-} / 02$ were markedly different in the normal and leukopenic groups (Table III). In the presence of normal numbers of circulating leukocytes, infection with the unencapsulated organism resulted in a three $\log _{10}$ decrease in bacterial concentrations within the CSF over the 18-h experimental time period when compared with the inoculum concentration of $\approx 10^{6.5} \mathrm{CFU}$. The leukopenic animals, however, were unable to clear the CSF of this unencapsulated strain (mean final concentration after $18 \mathrm{~h}, \log _{10}=6.67 \mathrm{CFU} / \mathrm{ml}$ ) and the CSF bacterial concentrations were significantly higher than the normal animals given $\mathrm{Rd}^{-} / \mathrm{b}^{-} / 02$.

The results of BBBP to circulating ${ }^{125} \mathrm{I}$-albumin in the different experimental groups is shown in Fig. 1. All infected animals had significantly increased BBBP when compared with their respective controls $(P<0.001) 18 \mathrm{~h}$ postinoculation. Control animals had uniformly low BBBP $(\leq 1.0 \%)$ with no significant differences seen between the normal and the leukopenic rats. Animals given the encapsulated organisms showed significant increases in BBBP in the normal animals compared with their leukopenic counterparts $(7.50 \pm 1.26$ vs. $4.75 \pm 0.37 \% ; P=0.04)$. BBBP in the normal animals challenged with the unencapsulated isogenic variant was uniformly low, with an average percent ${ }^{125} \mathrm{I}$-albumin concentration in the CSF of only $2.22 \pm 0.17 \%$. This low concentration of

Table III. CSF Bacterial Concentrations 18 h after Intracisternal Inoculation

\begin{tabular}{cccr}
\hline Inoculated strain & Normal rats* & Leukopenic rats* & $P$ value $^{*}$ \\
\hline $\mathrm{Rd}^{-} / \mathrm{b}^{+} / 02$ & $9.19 \pm 0.14^{\S}$ & $8.50 \pm 0.17^{\| 1}$ & 0.005 \\
$\mathrm{Rd}^{-} / \mathrm{b}^{-} / 02$ & $3.82 \pm 0.33^{\S}$ & $6.67 \pm 0.25^{\prime \prime}$ & $<0.001$ \\
\hline
\end{tabular}

\footnotetext{
* Mean \pm SEM $\log _{10} \mathrm{CFU} / \mathrm{ml} \mathrm{CSF}$.

₹ Student's $t$ test comparing normal rats with leukopenic rats challenged with either $\mathrm{Rd}^{-} / \mathrm{b}^{+} / 02$ or $\mathrm{Rd}^{-} / \mathrm{b}^{-} / 02$.

${ }^{\S} P<0.001$, normal rats challenged with $\mathrm{Rd}^{-} / \mathrm{b}^{+} / 02$ vs. $\mathrm{Rd}^{-} / \mathrm{b}^{-} / 02$.

" $P<0.001$, leukopenic rats challenged with $\mathrm{Rd}^{-} / \mathrm{b}^{+} / 02 \mathrm{vs}$. $\mathrm{Rd}^{-} / \mathrm{b}^{-} / 02$.
} 


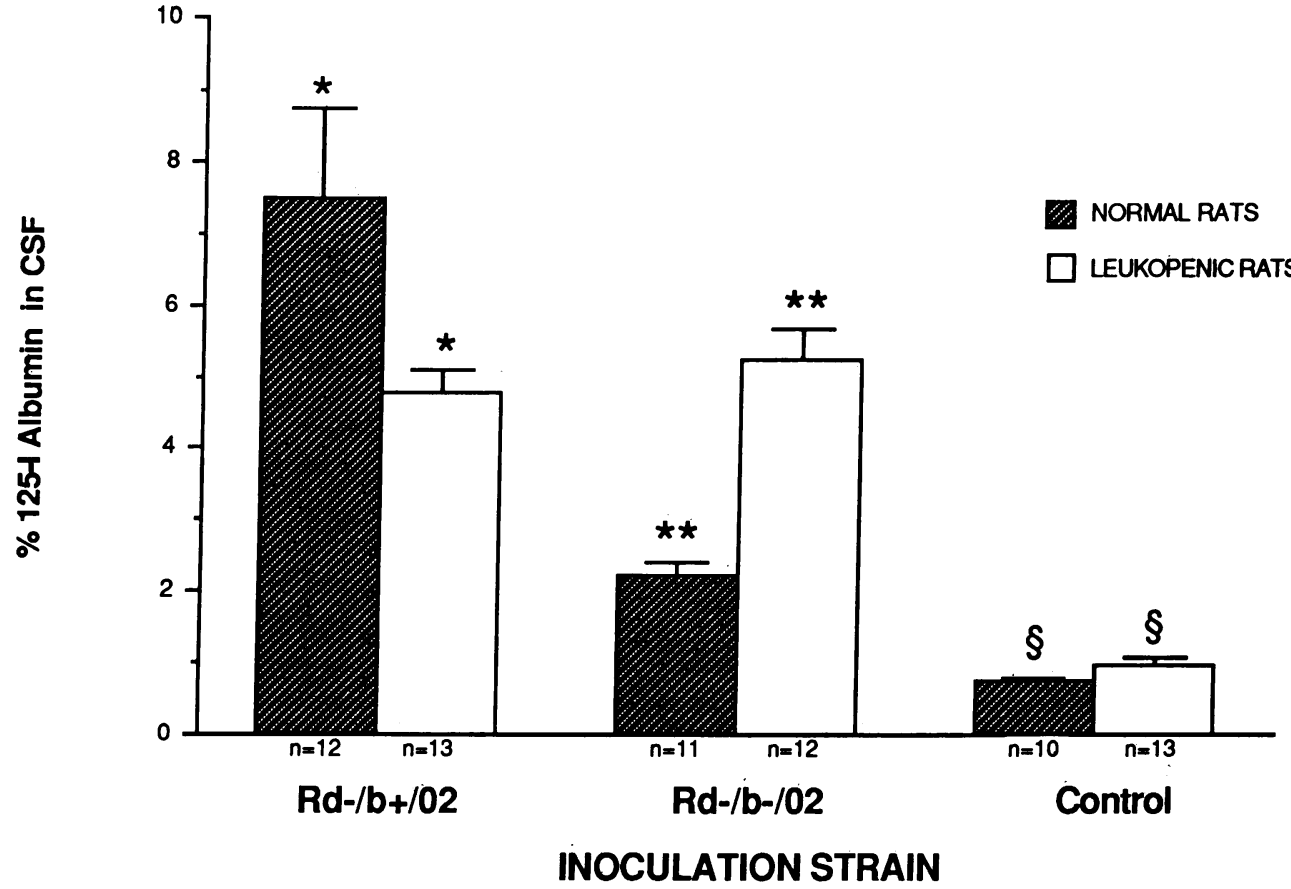

Figure 1. BBBP to circulating ${ }^{125} \mathrm{I}$-albumin $18 \mathrm{~h}$ after intracisternal challenge with encapsulated $\left(\mathrm{Rd}^{-} / \mathrm{b}^{+} / 02\right)$ or unencapsulated $\left(\mathrm{Rd}^{-} / \mathrm{b}^{-} / 02\right)$ isogenic mutants of $H$. influenzae or saline (control). Percent BBBP calculated as [cpm ${ }^{125} \mathrm{I}-\mathrm{CSF} / \mathrm{cpm}^{125} \mathrm{I}$ blood] $\times 100$. Values represent mean \pm SEM for normal and leukopenic animals. ${ }^{*} P=0.04 ;{ }^{* *} P$ $<0.001$; and $\S P=$ NS (Student's $t$ test, unpaired).
${ }^{125}$ I-albumin (i.e., BBBP only $\approx 2 \%$ after challenge with $\mathrm{Rd}^{-} / \mathrm{b}^{-} / 02$ ) was present despite the profound CSF pleocytosis (nearly 16,000 cells $/ \mathrm{mm}^{3}$ ) as shown in Table I. Depletion of circulating and CSF leukocytes resulted in increased BBBP (5.23 $\pm 0.44 \% ; P<0.001$ compared with the normal animals given the unencapsulated strain), approximating the BBBP values seen in the leukopenic animals challenged with the encapsulated organisms $(4.75 \pm 0.37$ vs. $5.23 \pm 0.44 \% ; P=0.41)$.

To assess the impact of organisms in the absence of replication, heat-killed $\mathrm{Rd}^{-} / \mathrm{b}^{+} / 02$ organisms were injected into the CSF in two concentrations. The low dose contained the standard inoculum of organisms $\left(\approx 10^{6.5} \mathrm{CFU}\right)$ and achieved a final CSF concentration of $\approx 10^{7.5} \mathrm{CFU} / \mathrm{ml}$, assuming CSF volume to be $\approx 100-150 \mu \mathrm{l} / \mathrm{rat}$. In an attempt to simulate the final 18-h concentration of the live $\mathrm{Rd}^{-} / \mathrm{b}^{+} / 02$ organism, 1.3 $\times 10^{8} \mathrm{CFU}$ heat-killed $\mathrm{Rd}^{-} / \mathrm{b}^{+} / 02$ was injected to achieve a final concentration of $\approx 10^{9} \mathrm{CFU} / \mathrm{ml} \mathrm{CSF}$. As shown in Table IV, intracisternal inoculation of low doses of dead $\mathrm{Rd}^{-} / \mathrm{b}^{+} / 02$ caused a massive CSF pleocytosis but resulted in little increase in BBBP. Leukopenia did not influence the degree of BBBP with this inoculum despite few leukocytes being present in the CSF. The use of higher concentrations of dead $\mathrm{Rd}^{-} / \mathrm{b}^{+} / 02$ resulted in a similar CSF concentration of leukocytes compared with the lower doses of dead organisms, but a significantly higher degree of BBBP was seen when compared with animals given the lower dose $(4.88 \pm 0.53$ vs. $1.79 \pm 0.25 \%, P$ $<0.001$ for the normal rats, and $3.53 \pm 0.30$ vs. $2.08 \pm 0.25 \%, P$ $=0.002$, for the leukopenic rats). As seen in the experiments with live organisms (Fig. 1), normal animals had significantly greater BBBP than leukopenic rats challenged with the high dose of heat-killed $\mathrm{Rd}^{-} / \mathrm{b}^{+} / 02(P=0.05)$.

The results of the experiments with live $\mathrm{Rd}^{-} / \mathrm{b}^{+} / 02$ were compared to a pathologic isolate of $H$. influenzae type $b$ frequently used in other models of experimental meningitis, strain Eagan (which was also the donor strain for the recombinants [see Methods]). The same trends were seen in rats inocu- lated with the Eagan strain as were seen with $\mathrm{Rd}^{-} / \mathrm{b}^{+} / 02$ and were similar to our results reported previously (14). Experimental meningitis induced with the Eagan strain produced lower bacterial concentrations in CSF and blood and correspondingly smaller increases in BBBP when compared with $\mathrm{Rd}^{-} / \mathrm{b}^{+} / 02$. For example, the mean \pm SEM bacterial concentrations $\left(\log _{10} \mathrm{CFU} / \mathrm{ml}\right)$ in blood and CSF were $4.90 \pm 0.15$ and $8.41 \pm 0.12$ vs. $6.43 \pm 0.37$ and $9.19 \pm 0.14$ after challenge with strain Eagan and $\mathrm{Rd}^{-} / \mathrm{b}^{+} / 02$, respectively. The mean $\pm \mathrm{SEM}$ BBBP to circulating albumin in normal rats was $6.49 \pm 0.74$ vs. $7.50 \pm 1.26 \%$ after inoculation of Eagan and $\mathrm{Rd}^{-} / \mathrm{b}^{+} / 02$. Significantly decreased BBBP $(P=0.05)$ was again seen in leukopenic rats compared with normal rats inoculated with the Eagan strain. Additionally, CSF bacterial concentrations tended to be higher in the normal group compared with the leukopenic group (mirroring the differences seen with $\left.\mathrm{Rd}^{-} / \mathrm{b}^{+} / 02\right)$ but did not attain statistical significance $(P=0.18)$ (data not shown).

Table IV. BBBP and CSF Leukocyte Response to Challenge with Heat-killed $\mathrm{Rd}^{-} / \mathrm{b}^{+} / 02$

\begin{tabular}{cccccc}
\hline & \multicolumn{2}{c}{ CSF WBC concentration* } & & \multicolumn{2}{c}{ BBBP* $^{*}$} \\
\cline { 2 - 3 } \cline { 5 - 6 } Inocula & $\begin{array}{c}\text { Normal } \\
\text { rats }\end{array}$ & $\begin{array}{c}\text { Leukopenic } \\
\text { rats }\end{array}$ & & $\begin{array}{c}\text { Normal } \\
\text { rats }\end{array}$ & $\begin{array}{c}\text { Leukopenic } \\
\text { rats }\end{array}$ \\
\hline Low $\approx 10^{6.5} \mathrm{CFU}$ & $\begin{array}{c}29.9 \pm 9.7 \\
(n=8)\end{array}$ & $\begin{array}{c}0.06 \pm 0.02 \\
(n=14)\end{array}$ & & $\begin{array}{c}1.79 \pm 0.25^{8} \\
(n=8)\end{array}$ & $\begin{array}{c}2.08 \pm 0.25^{8} \\
(n=14)\end{array}$ \\
High $\approx 10^{8.5} \mathrm{CFU}$ & $\begin{array}{c}20.7 \pm 3.2 \\
(n=8)\end{array}$ & $\begin{array}{c}0.03 \pm 0.01 \\
(n=7)\end{array}$ & $\begin{array}{c}4.88 \pm 0.53^{\prime \prime} \\
(n=8)\end{array}$ & $\begin{array}{c}3.53 \pm 0.30^{\prime \prime} \\
(n=7)\end{array}$ \\
\hline
\end{tabular}

* Mean \pm SEM leukocytes $\times 10^{3} / \mathrm{mm}^{3} \mathrm{CSF}$.

$\ddagger$ Mean \pm SEM cpm CSF/cpm blood $\times 100$, expressed as percent.

$P=$ NS.

" $P=0.05$. 


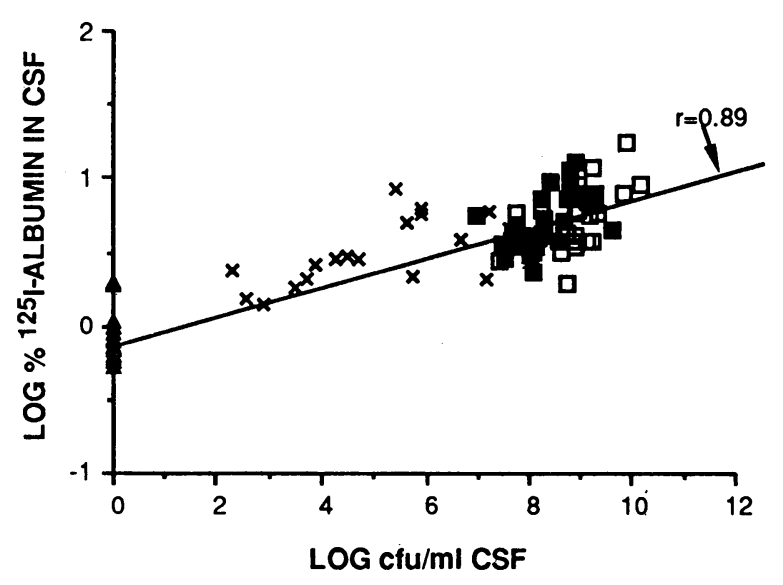

Figure 2. Relationship between CSF concentrations of $H$. influenzae and BBBP during experimental meningitis. Bacterial concentrations represent data from all animals given $\mathrm{Rd}^{-} / \mathrm{b}^{+} / 02(\square), \mathrm{Rd}^{-} / \mathrm{b}^{-} / 02(X)$, Eagan ( () , and saline (control) $(\Delta)(n=98)$, and are plotted as a $\log _{10}$ $\mathrm{CFU} / \mathrm{ml} \mathrm{CSF}$ with control animals assigned a concentration of zero. BBBP was calculated as in Fig. 1 and expressed as the $\log _{10} \%$ BBBP. The line represents a best-fit simple regression analysis with $r=0.89$, $P<0.001$.

Because the alterations in BBBP appeared to parallel the changes in CSF concentrations of bacteria, the relationship between live CSF bacterial concentrations $\left(\mathrm{Rd}^{-} / \mathrm{b}^{+} / 02\right.$, $\mathrm{Rd}^{-} / \mathrm{b}^{-} / 02$, and Eagan) and the percent albumin in the CSF was examined (Fig. 2). The percent penetration of ${ }^{125} \mathrm{I}$-albumin expressed as a log to the base 10 was plotted versus bacterial concentrations so that the correlation would be linear rather than exponential, and control animals were assigned a value of zero for bacterial concentration. The correlation $(r$ $=0.89 ; n=98 ; P<0.001)$ suggests that BBBP was markedly influenced by bacterial concentrations within the CSF.

\section{Discussion}

Utilizing a model of meningitis in the leukopenic rat that allowed quantitative assessment of BBB injury, we have shown that CSF leukocytes can augment changes in BBBP. CSF leukocytes (and peripheral blood leukocytes) were incapable of controlling the replication of the type b encapsulated strain of $H$. influenzae. The bacterial surface structure that governed this resistance to host clearance appeared to be the type $b$ capsular polysaccharide. Unencapsulated bacteria were cleared by CSF leukocytes in normal rats but were not cleared in the absence of CSF leukocytes, suggesting that phagocytosis is the major function of these leukocytes, but the presence of capsule effectively inhibits this role. Heat-killed $\mathrm{Rd}^{-} / \mathrm{b}^{+} / 02$ resulted in a massive rise in CSF leukocytes but alterations in BBBP were primarily dependent on bacterial concentrations. Although no clear correlation between CSF pleocytosis and BBBP was observed, the present data suggest that the BBB injury induced by the CSF leukocytes is one of augmentation of permeability (at least after challenge with $\mathrm{Rd}^{-} / \mathrm{b}^{+} / 02$ and Eagan) and that a major determinant of BBBP $18 \mathrm{~h}$ after intracisternal inoculation is CSF bacterial concentrations.

Measurement of injury to the CNS during bacterial meningitis is difficult to quantitate. While several markers of injury exist (CSF leukocytes, decreased glucose concentration, lactic acid production, brain edema, and the physiologic parameters of temperature, hemodynamics, and death), few deal with a quantitative assessment of the physiologic dynamics within the CNS. Normal integrity of this immunologically impaired compartment is maintained by the $\mathrm{BBB}$ which mainly resides in the unique physiology of the cerebral capillary endothelium. These capillaries differ from systemic capillaries in their marked reduction of pinocytotic vesicles, absence of fenestrations, and the presence of tight junctional complexes joining adjacent endothelial cells $(21,22)$. Previous work from our laboratory (14) has recently shown that morphologic changes occur temporally during experimental bacterial meningitis with an increase in pinocytotic vesicle formation and separation of tight junctional complexes as the disease progressed. These morphologic changes were paralleled by increasing concentrations of circulating ${ }^{125} \mathrm{I}$-labeled albumin in the CSF after intravenous administration. The use of a labeled protein allows an accurate measurement of increased BBBP (a functional parameter of BBB injury) and correlated well with the observed morphologic changes in the cerebral microvascular endothelium. Injury to this crucial component of CNS homeostasis is therefore more easily and accurately measured by this physiologic technique and provides a useful tool in assessing the role of both meningeal pathogens and host factors in the development and progression of bacterial meningitis, and the pathophysiologic consequences of the experimental infection.

While the results of our experiments suggest that leukocytes can augment damage to the BBB during meningitis, the data indicate that bacterial concentrations in the CSF play a major role. Several studies have shown that the clinical outcomes (morbidity and mortality) of meningitis are influenced by the CSF concentration of bacteria $(23,24)$. This appears to be the first experimental observation that correlates the degree of injury to the BBB with the number of bacteria present within the CSF. Although leukocytes may alter BBBP, the major factor appeared to be the bacterial concentrations within the CSF. Whether these changes are due to the presence of the entire bacteria or are closely related to one or more bacterial components (as is the case with pneumococci and pneumococcal cell wall [25]) is actively under investigation at this time. Preliminary work from Syrogiannopoulos and others (26) has shown that the LOS of $H$. influenzae type $b$ is extraordinarily potent in inducing a CSF pleocytosis at nanogram concentrations and may be one of the active components in the altered BBBP observed in our model. Indeed, alterations in the LOS content between Eagan and the transformed strains may explain part of the differences in the degree of BBBP seen between those groups. The results of our experiments with inactivated $\mathrm{Rd}^{-} / \mathrm{b}^{+} / 02$ suggest that the CSF concentration of the bacteria (or their subcomponents) can lead to marked BBB alterations on a concentration dependent basis.

Several other important observations regarding the host parasite interaction during bacterial meningitis were noted in this study. First, it was shown that BBBP can be significantly altered in the near absence of CSF leukocytes. Early work by Harter and Petersdorf had suggested that significant ". . . changes in the permeability of the blood-cerebrospinal fluid barrier in this situation [leukopenic dogs with experimental pneumococcal meningitis] are probably of minor consequence (3)." The ability to quantitate the degree of injury and determine whether or not leukocytes played a role, however, was not addressed in that review. Ernst and colleagues found, to 
the contrary, that CSF protein increased markedly in the leukopenic lapine model of pneumococcal meningitis but could not comment on the effect of leukocytes because of the large variation (tenfold) in CSF protein concentrations (27). This may reflect the fact that total CSF protein does not result solely from the transudation of serum proteins across the damaged $\mathrm{BBB}$; proteins generated within the CSF during the injury also contribute to total protein concentration. Our study has shown that the BBB is, in fact, significantly altered during meningitis in the near absence of CSF leukocytes.

While the appearance of a totally serum-derived marker in the CSF of leukopenic animals suggests that breakdown of the barrier does not depend on leukocytes, other explanations for our results are also possible. Kitchens has demonstrated that endothelium can undergo changes associated with thrombocytopenia (28) although those changes probably do not account for the findings in our present experiments. Thrombocytopenia, in that work (28), induced alterations in the peripheral venules of the tongue (vasculature that is quite different from the cerebral capillary endothelium) and only occurred at platelet concentrations below $5,000 / \mathrm{mm}^{3}$, far lower concentrations than were seen with our experiments. Additionally, while secondary influences of cyclophosphamide treatment may have directly altered BBB function, the more likely conclusion (especially with similar control groups) suggests that leukocytes were not essential to produce damage to the BBB over the time course of the experiments, but $(a)$ were able to enhance the degree of BBB injury from the infectious process itself, and $(b)$ were potentially deleterious to the host.

Similar conclusions were drawn from Petersdorf's earlier work (11), which suggested that leukopenic animals with experimental pneumococcal meningitis had slightly increased survival times when compared with their normal counterparts. Scheld et al. determined that methylprednisolone given to rabbits with pneumococcal meningitis decreased CSF outflow resistance during meningitis; one presumed mechanism for this decreased resistance could be attributed to the antiinflammatory effects of the corticosteroids (7). Tauber and colleagues (8) found that administration of dexamethasone alone decreased brain water content, CSF pressure, and lactate production in rabbits with experimental pneumococcal meningitis compared with rabbits given no treatment. Ampicillin therapy was able to reverse CSF pressure and brain water alterations associated with this model of experimental meningitis but was unable to reverse CSF lactate levels. Those observations have been applied to human trials and have preliminarily shown that the use of dexamethasone with antibiotic therapy of bacterial meningitis in infants and children has decreased the incidence of associated deafness while not affecting mortality (29). If the primary role of these interventions (steroids $[7,8,29]$ ) were related primarily to alterations in leukocyte function, then manipulation of leukocyte function may have far reaching effects on the concurrent therapy of bacterial meningitis. This model would provide a means of studying the influence of leukocytes (and leukocyte mediators) on BBB function and should be useful in establishing potential therapeutic adjuncts.

Intuitively, it would be expected that interference with leukocyte function during meningitis might be detrimental. During infection in this area of altered host resistance one would presume that a fully functional leukocyte would be essential in controlling this closed-space infection. This and other experiments, however, reveal that the leukocyte cannot properly control $H$. influenzae type b. Rubin et al. have previously shown that $H$. influenzae type $\mathrm{b}$ is capable of intravascular replication in the normal rat (30). Since this organism can grow even in the presence of a full complement of host defenses in the peripheral circulation it is not surprising that the influx of leukocytes into the CSF would be ineffective in halting bacterial replication. Our experiments confirmed these observations as normal animals had similar, if not higher, concentrations of the encapsulated organism in the CSF and blood when compared with leukopenic rats. Hence, the contribution of leukocytes may not be entirely advantageous during meningitis with encapsulated $H$. influenzae type b.

Leukocytes do, however, appear to play a major role in the CSF when challenged with organisms that cannot easily evade host clearance mechanisms. In work by Chow et al. (31), Candida albicans was readily cleared from the CSF of normal dogs after intracisternal challenge. Leukopenic dogs given the same organism were unable to control the fungus and died. In our study, $\mathrm{Rd}^{-} / \mathrm{b}^{-} / 02$ was rapidly cleared and resulted in lower bacterial concentrations in the CSF and blood in normal rats when compared with its isogenic encapsulated counterpart. Decreasing the number of leukocytes resulted in the increased CSF concentrations of the unencapsulated strain with occasional bacteremia. Indeed, when the organisms were not cleared from the CSF of the leukopenic rats, the degree of altered BBBP was similar to that observed in leukopenic animals challenged with the encapsulated strain. Leukocytes therefore are effective in the clearance of some types of organisms from the CSF.

Whether the suppression of leukocyte function will be advantageous in all forms of bacterial meningitis caused by encapsulated organisms is not addressed in this study. While leukocytes are not effective in the clearance of $H$. influenzae type $b$ from the CSF and bloodstream, their role in the control of other encapsulated organisms is not well defined. A previous study showed that while $H$. influenzae type b can escape host clearance and replicate within the vascular space, $S$. pneumoniae, a common meningeal pathogen, could not replicate within the intravascular space and required extravascular sites for the development of sustained bacteremia (30). Ernst has shown in leukopenic rabbits with pneumococcal meningitis that the CSF concentration of bacteria was unaffected by the leukopenic state, but that the blood concentrations were $\sim 100$-fold higher (27). Although the numbers were small and differ from Petersdorf's original observations (11), it raises questions concerning the applicability of leukocyte alteration to all forms of bacterial meningitis. Future studies on the role of therapeutic adjuncts should be stratified to include this possibility.

Multiple attempts at a nonbacterial model of sterile pleocytosis (mucin [6], C5,$f$-Met-Leu-Phe [32], and alpha bungarotoxin [33]) to address the impact of leukocytes alone were all unsuccessful in this model. It is evident, however, that leukocytes, even in massive CSF concentrations, did not appear to correlate with the observed changes in BBBP at the time point assessed, as shown in the experiments employing dead organisms.

These and other experiments do not address the observations that bacterial meningitis is an unusual disease in the leukopenic patient [34]. The exact pathogenic mechanisms for the initiation of bacterial meningitis are not known, but it can be concluded from clinical observations that the mere absence 
of leukocytes is not the major pathogenic mechanism in bacterial meningitis. This work and the work of others using leukopenic animal models does suggest that "nonpathogenic" bacteria and fungi can cause meningitis in the near absence of CSF leukocytes after intracisternal challenge. The absence of leukocytes however, probably does not alter the access of organisms to the CSF, resulting in no increased incidence of bacterial meningitis during profound leukopenia secondary to immunosuppressive therapy in humans.

In conclusion, these experiments have quantitated the alterations in BBBP during experimental $H$. influenzae meningitis after challenge with isogenic strains. The degree of the alterations in BBBP $18 \mathrm{~h}$ after intracisternal inoculation are more dependent on the CSF concentration of bacteria than on the CSF leukocyte concentrations. Leukocytes can augment the changes in BBBP after challenge with encapsulated $H$. influenzae but are not necessary to cause damage. Whether whole bacteria or their subcellular components mediate the changes seen are not known at the present time. Since increasing BBBP was seen with increasing concentrations of heat-killed bacteria in the CSF, it is apparent that the organisms can induce changes without being capable of replication. Future work to investigate which bacterial components mediate these changes and the mechanisms of BBB injury are currently under way in our laboratory.

\section{Acknowledgments}

The authors thank Vincent J. Quagliarello, M.D., for his invaluable assistance.

This work was supported in part by research grants from the National Institutes of Allergy and Infectious Diseases (AI 17904), and by the Kate Miller Jeffress and Thomas Jeffress Memorial Trust. W.M.S. is an Established Investigator of the American Heart Association. A.J.L. was supported by a National Institutes of Health Training Grant (T32-AI-07046).

\section{References}

1. Swartz, M. N., and P. R. Dodge. 1965. Bacterial meningitis: a review of selected aspects. N. Engl. J. Med. 272:725-731.

2. Schlech, W. F., J. I. Ward, J. D. Band, A. Hightower, D. W. Fraser, and C. V. Broome. 1985. Bacterial meningitis in the United States, 1978 through 1981 . The national bacterial meningitis surveillance study. (JAMA) J. Am. Med. Assoc. 253:1749-1754.

3. Harter, D. H., and R. G. Petersdorf. 1960. A consideration of the pathogenesis of bacterial meningitis: review of experimental and clinical studies. Yale J. Biol. Med. 32:280-309.

4. McAllister, C. K., J. M. O'Donoghue, and H. N. Beaty. 1975. Experimental pneumococcal meningitis. II. Characterization and quantitation of the inflammatory process. J. Infect. Dis. 132:355-360.

5. Sears, M. R., J. M. O'Donoghue, H. K. Fisher, and H. N. Beaty. 1974. Effect of experimental pneumococcal meningitis on respiration and circulation in the rabbit. J. Clin. Invest. 54:18-23.

6. Nolan, C. M., C. K. McAllister, E. Walters, and H. N. Beaty. 1978. Experimental pneumococcal meningitis. IV. The effect of methyl prednisolone on meningeal inflammation. J. Lab. Clin. Med. 91:979-988.

7. Scheld, W. M., R. G. Dacey, H. R. Winn, J. E. Welsh, J. A. Jane, and $M$. A. Sande. 1980. Cerebrospinal fluid outflow resistance in rabbits with experimental meningitis. Alterations with penicillin and methylprednisolone. J. Clin. Invest. 66:243-253.

8. Tauber, M. G., H. Khayam-Bashi, and M. A. Sande. 1985.
Effects of ampicillin and corticosteroids on brain water content, cerebrospinal fluid pressure, and cerebrospinal fluid lactate levels in experimental pneumococcal meningitis. J. Infect. Dis. 151:528-534.

9. Fishman, R. A., K. Sligar, and R. B. Hake. 1977. Effects of leukocytes on brain metabolism in granulocytic brain edema. Ann. Neurol. 2:89-94.

10. Giampaolo, C., M. Scheld, J. Boyd, J. Savory, M. Sande, and M. Wills. 1980. Leukocyte and bacterial interrelationships in experimental meningitis. Ann. Neurol. 9:328-333.

11. Petersdorf, R. G., and C. N. Luttrell. 1962. Studies on the pathogenesis of meningitis. I. Intrathecal infection. J. Clin. Invest. 41:311-319.

12. Simberkoff, M. S., N. H. Moldover, and J. J. Rahal. 1980. Absence of detectable bactericidal and opsonic activities in normal and infected human cerebrospinal fluids. A regional host defense deficiency. J. Lab. Clin. Med. 95:362-372.

13. Zwahlen, A., U. E. Nydegger, P. Vaudaux, P. Lambert, and F. A. Waldvogel. 1982. Complement-mediated opsonic activity in normal and infected human cerebrospinal fluid: early response during bacterial meningitis. J. Infect. Dis. 145:635-646.

14. Quagliarello, V. J., W. J. Long, and W. M. Scheld. 1986. Morphologic alteration of the blood-brain barrier with experimental meningitis in the rat. Temporal sequence and role of encapsulation. J. Clin. Invest. 77:1084-1095.

15. Zwahlen, A., L. G. Rubin, and E. R. Moxon. 1986. Contribution of lipopolysaccharide to pathogenicity of Haemophilus influenzae: comparative virulence of genetically-related-strains in rats. $\mathrm{Mi}$ crobial. Pathogen. 1:465-473.

16. Pepple, J., E. R. Moxon, and R. H. Yolken. 1980. Indirect enzyme-linked immunosorbent assay for the quantitation of the typespecific antigen of Haemophilus influenzae b: a preliminary report. $J$. Pediatr. 97:233-237.

17. Hoiseth, S. K., C. J. Connelly, and E. R. Moxon. 1985. Genetics of spontaneous, high-frequency loss of b capsule expression in Haemophilus influenzae. Infect. Immun. 49:389-395.

18. Zwahlen, A., J. A. Winckelstein, and E. R. Moxon. 1983. Surface determinants of Haemophilus influenzae pathogenicity: comparative virulence of capsular transformants in normal and complement-depleted rats. J. Infect. Dis. 148:385-394.

19. Kimura, A., and E. J. Hansen. 1986. Antigenic and phenotypic variations of Haemophilus influenzae type b lipopolysaccharide and their relationship to virulence. Infect. Immun. 51:69-79.

20. Kimura, A., C. C. Patrick, E. E. Miller, L. D. Cope, G. H. McCracken, Jr., and E. J. Hansen. 1987. Haemophilus influenzae type b lipooligosaccharide: stability of expression and association with virulence. Infect. Immun. 55:1979-1986.

21. Reese, T. S., and M. J. Karnovsky. 1967. Fine structural localization of a blood brain barrier to exogenous peroxidase. J. Cell Biol. 34:207-217.

22. Brightman, M. W., and T. S. Reese. 1969. Junctions between intimately opposed cell membranes in the vertebrate brain. J. Cell Biol. 40:648-677.

23. Feldman, W. E. 1977. Relation of concentrations of bacteria and bacterial antigen in cerebrospinal fluid to prognosis in patients with bacterial meningitis. N. Engl. J. Med. 296:433-435.

24. Feldman, W. E., C. M. Ginsburg, G. H. McCracken, Jr., D. Allen, P. Ahmann, J. Graham, and L. Graham. 1982. Relation of concentrations of Haemophilus influenzae type $\mathrm{b}$ in cerebrospinal fluid to late sequelae of patients with meningitis. J. Pediatr. 100:209212.

25. Tuomanen, E., H. Liu, B. Hengstler, O. Zak, and A. Tomasz. 1985. The induction of meningeal inflammation by components of the pneumococcal cell wall. J. Infect. Dis. 151:859-868.

26. Syrogiannopoulos, G. A., E. J. Hansen, A. L. Erwin, R. S. Munford, and G. H. McCracken, Jr. 1986. The role of Haemophilus influenzae type b lipopolysaccharide (LPS) in the induction of meningeal inflammation. In Program and Abstracts of the 26th Interscience 
Conference on Antimicrobial Agents and Chemotherapy. Abstract No. 747.

27. Ernst, J. D., J. M. Decazes, and M. A. Sande. 1983. Experimental pneumococcal meningitis: role of leukocytes in pathogenesis. Infect. Immun. 42:275-279.

28. Kitchen, C. S., and L. Weiss. 1975. Ultrastructural changes of endothelium associated with thrombocytopenia. J. Clin. Invest. 46:567-578.

29. Syrogiannopoulos, G. A., B. J. Freij, K. D. Olsen, and G. H. McCracken. 1986. Dexamethasone treatment for bacterial meningitis in infants and children. In Program and Abstracts of the 26th Interscience Conference on Antimicrobial Agents and Chemotherapy. Abstract No. 868.

30. Rubin, L. G., A. Zwahlen, and E. R. Moxon. 1985. Role of intravascular replication in the pathogenesis of experimental bacteremia due to Haemophilus influenzae type b. J. Infect. Dis. 152:307-314.

31. Chow, H. S., S. C. Sarpel, and R. B. Epstein. 1980. Pathophysiology of Candida albicans meningitis in normal, neutropenic, and granulocyte transfused dogs. Blood. 55:546-551.

32. Tauber, M. G., and M. A. Sande. 1984. Leukocytes mediate brain edema in experimental meningitis. Clin. Res. 32:559a. (Abstr.)

33. Dermietzel, R., and P. M. Faustman. 1985. Extravasation of polymorphonuclear leukocytes from cerebral microvasculature. Inflammatory response induced by alpha bungarotoxin. Cell Tissue Res. 242:399-407.

34. Lukes, S. A., J. B. Posner, S. Nielsen, and D. Armstrong. 1984. Bacterial infections of the CNS in neutropenic patients. Neurol. Cleveland. 34:269-275. 